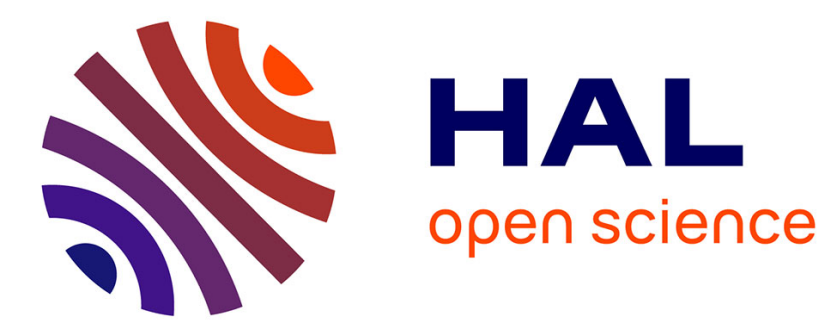

\title{
Tight bounds for rational sums of squares over totally real fields \\ Ronan Quarez
}

\section{To cite this version:}

Ronan Quarez. Tight bounds for rational sums of squares over totally real fields: Tight bounds for rational sums of squares over totally real fields. Rendiconti del Circolo Matematico di Palermo, 2010, 59 (3), pp.377-388. hal-00403920

\section{HAL Id: hal-00403920 \\ https://hal.science/hal-00403920}

Submitted on 14 Jul 2009

HAL is a multi-disciplinary open access archive for the deposit and dissemination of scientific research documents, whether they are published or not. The documents may come from teaching and research institutions in France or abroad, or from public or private research centers.
L'archive ouverte pluridisciplinaire HAL, est destinée au dépôt et à la diffusion de documents scientifiques de niveau recherche, publiés ou non, émanant des établissements d'enseignement et de recherche français ou étrangers, des laboratoires publics ou privés. 


\title{
BOUNDING THE RATIONAL SUMS OF SQUARES OVER TOTALLY REAL FIELDS
}

\author{
RONAN QUAREZ
}

\begin{abstract}
Let $K$ be a totally real Galois number field. C. J. Hillar proved that if $f \in \mathbb{Q}\left[x_{1}, \ldots, x_{n}\right]$ is a sum of $m$ squares in $K\left[x_{1}, \ldots, x_{n}\right]$, then $f$ is a sum of $N(m)$ squares in $\mathbb{Q}\left[x_{1}, \ldots, x_{n}\right]$, where $N(m) \leq 2^{[K: \mathbb{Q}]+1} \cdot(\underset{2}{[K: \mathbb{Q}]+1}) \cdot 4 m$, the proof being constructive.

We show in fact that $N(m) \leq(4[K: \mathbb{Q}]-3) \cdot m$, the proof being constructive as well.
\end{abstract}

\section{INTRODUCTION}

In the theory of semidefinite linear programming, there is a question by Sturmfels Question 1.1 (Sturmfels). If $f \in \mathbb{Q}\left[x_{1}, \ldots, x_{n}\right]$ is a sum of squares in $\mathbb{R}\left[x_{1}, \ldots, x_{n}\right]$, then is $f$ also a sum of squares in $\mathbb{Q}\left[x_{1}, \ldots, x_{n}\right]$ ?

Hillar ([3]) answers the question in the case where the sum of squares has coefficients in a totally real Galois number field :

Theorem 1.2 (Hillar). Let $f \in \mathbb{Q}\left[x_{1}, \ldots, x_{n}\right]$ be a sum of $m$ squares in $K\left[x_{1}, \ldots, x_{n}\right]$ where $K$ is a totally real Galois extension of $\mathbb{Q}$. Then, $f$ is a sum of

$$
{ }_{2}[K: \mathbb{Q}]+1 \cdot\left(\begin{array}{c}
{[K: \mathbb{Q}]+1} \\
2
\end{array}\right) \cdot 4 m
$$

squares in $\mathbb{Q}\left[x_{1}, \ldots, x_{n}\right]$.

The aim of this note is to show, modifying a little bit Hillar's proof, that only $(4[K: \mathbb{Q}]-3) \cdot m$ squares are needed (that is Theorem 3.1). Moreover, as in [3], the argument is constructive.

\section{HiLLAR'S METHOD}

Having in mind the Lagrange's four squares Theorem, we focus ourselves on rational sum of squares i.e. linear combination of squares with positive rationnal coefficients.

Let $K$ be a totally real Galois extension of $\mathbb{Q}$ which we write $K=\mathbb{Q}(\theta)$ with $\theta$ a real algebraic number, all of whose conjugates are also real. We set $r=[K: \mathbb{Q}]$ and $G=\operatorname{Gal}(K / \mathbb{Q})$.

Let $f \in \mathbb{Q}\left[x_{1}, \ldots, x_{n}\right]$ be a sum of $m$ squares in $K\left[x_{1}, \ldots, x_{n}\right]$, namely $f=$ $\sum_{k=1}^{m} f_{k}^{2}$, with $f_{k} \in K\left[x_{1}, \ldots, x_{n}\right]$. Summing over all actions of $G$ (i.e. "averaging"), we get

Date: July 14, 2009.

2000 Mathematics Subject Classification. 12Y05, 12F10, 11E25, $13 \mathrm{~B} 24$.

Key words and phrases. Rational sum of squares, semidefinite programming, totally real number field. 


$$
f=\frac{1}{|G|} \sum_{k=1}^{m} \sum_{\sigma \in G}\left(\sigma f_{k}\right)^{2}
$$

Next, we write each $f_{k}$ in the form

$$
f_{k}=\sum_{i=0}^{r-1} q_{i} \theta^{i}
$$

where $q_{i} \in \mathbb{Q}\left[x_{1}, \ldots, x_{n}\right]$. Then,

$$
\sum_{\sigma \in G}\left(\sigma f_{k}\right)^{2}=\sum_{j=1}^{r}\left(\sum_{i=0}^{r-1} q_{i}\left(\sigma_{j} \theta\right)^{i}\right)^{2}
$$

We may write this sum of squares as the following product of matrices

$$
\left(\begin{array}{c}
q_{0} \\
\vdots \\
q_{r-1}
\end{array}\right)^{T}\left(\begin{array}{cccc}
1 & \sigma_{1} \theta & \ldots & \left(\sigma_{1} \theta\right)^{r-1} \\
\vdots & \vdots & \ddots & \vdots \\
1 & \sigma_{r} \theta & \ldots & \left(\sigma_{r} \theta\right)^{r-1}
\end{array}\right)^{T}\left(\begin{array}{cccc}
1 & \sigma_{1} \theta & \ldots & \left(\sigma_{1} \theta\right)^{r-1} \\
\vdots & \vdots & \ddots & \vdots \\
1 & \sigma_{r} \theta & \ldots & \left(\sigma_{r} \theta\right)^{r-1}
\end{array}\right)\left(\begin{array}{c}
q_{0} \\
\vdots \\
q_{r-1}
\end{array}\right)
$$

We obtain what is called a Gram matrix ( $\mathrm{cf}$ [1]) associated to the sum of squares in (2). Let

$$
G=\left(\begin{array}{cccc}
1 & \sigma_{1} \theta & \ldots & \left(\sigma_{1} \theta\right)^{r-1} \\
\vdots & \vdots & \ddots & \vdots \\
1 & \sigma_{r} \theta & \ldots & \left(\sigma_{r} \theta\right)^{r-1}
\end{array}\right)^{T}\left(\begin{array}{cccc}
1 & \sigma_{1} \theta & \ldots & \left(\sigma_{1} \theta\right)^{r-1} \\
\vdots & \vdots & \ddots & \vdots \\
1 & \sigma_{r} \theta & \ldots & \left(\sigma_{r} \theta\right)^{r-1}
\end{array}\right)
$$

Note that the entries of $G$ are in $\mathbb{Q}$ since they are invariant under the $\sigma_{j}$ 's.

Now, we come to the slight modification of the proof of Hillar that will improve the bound.

\section{LU-DeCOMposition OF the Gram Matrix}

If $u(x)$ denotes the minimal polynomial of the Galois extension $K$ over $\mathbb{Q}$, then the $(i, j)$-th entry of the matrix $G$ is the $i+j-2$-th Newton sum of $\left(\sigma_{1}, \ldots, \sigma_{r}\right)$ the roots of $u(x)$. It is well known that the rank of $G$ is equal to $r$ and its signature (the difference between the positive eigenvalues and the negative ones) is equal to the number of real roots of $u(x)$ (see for instance [2, Theorem 4.57]). In our case, we readily deduce that $G$ is a positive definite matrix since $K$ is totally real. Thus, all its principal minors are different from zero (they are strictly positive !) and $G$ admits a LU-decomposition which we may put in a symmetric form

$$
G=U^{T} D U
$$

where $D$ is diagonal and $U$ is upper triangular with diagonal identity, and $U, D$ have rational entries.

We may view this decomposition as a matricial realization of the Gauss algorithm which reduce the quadratic form given by $G$. 
Now, if we denote by $f_{1}, \ldots, f_{r}$ the polynomials in $\mathbb{Q}\left[x_{1}, \ldots, x_{r}\right]$ appearing as the rows of the matrix $U \times\left(\begin{array}{c}q_{0} \\ \vdots \\ q_{r-1}\end{array}\right)$ and by $d_{1}, \ldots, d_{r}$ the rational entries onto the diagonal of $D$, then we get from (2) the identity :

$$
\frac{1}{|G|} \sum_{\sigma \in G}\left(\sigma f_{k}\right)^{2}=\frac{d_{1}}{|G|} g_{1}^{2}+\ldots+\frac{d_{r}}{|G|} g_{r}^{2}
$$

This construction leads to

Theorem 3.1. Let $f \in \mathbb{Q}\left[x_{1}, \ldots, x_{n}\right]$ be a sum of $m$ squares in $K\left[x_{1}, \ldots, x_{n}\right]$, where $K$ is a totally real Galois extension of $\mathbb{Q}$. Then, $f$ is a sum of $(4[K: \mathbb{Q}]-3) \cdot m$ squares in $\mathbb{Q}\left[x_{1}, \ldots, x_{n}\right]$.

Proof. By (1) and (3), it suffices to apply Lagrange's four squares Theorem to get that $f$ is a sum of $4[K: \mathbb{Q}] \cdot m$ squares in $\mathbb{Q}\left[x_{1}, \ldots, x_{n}\right]$.

But let us note that the first diagonal entry of $D$ is always $d_{1}=r=[K: \mathbb{Q}]$. Then, by the averaging process the first coefficient appearing in the rational sum of squares in (3) is $\frac{d_{1}}{|G|}=1$ : already a square in $\mathbb{Q}$ ! Whereas the others coefficients $\frac{d_{i}}{|G|}$ in the rational sum of squares could be any positive rational which we rewrite as a sum of 4 squares. This concludes the proof.

Remark 3.2. Beware that if we perform the Cholesky algorithm to the matrix $G$ instead of the LU-decomposition, it yields a factorisation $G=U^{T} U$ where $U$ is lower triangular but with entries in $\mathbb{Q}\left[\sqrt{d_{1}}, \ldots, \sqrt{d_{r}}\right]$ for some integers $d_{1}, \ldots, d_{r}$. Then, an averaging argument would produce identities over $\mathbb{Q}$ but will raise the number of squares by an unexpected multiplicative factor $2^{[K: \mathbb{Q}]}$.

Let us consider as an example, the simple case of quadratic extensions :

Example 3.3. Let $K=\mathbb{Q}(\sqrt{d})$ where $d \in \mathbb{Q}$ is not a square. The extension $K$ is always Galois, and it is totally real if $d \geq 0$.

Let $f \in \mathbb{Q}\left(x_{1}, \ldots, x_{n}\right)$ be such that $f=\sum_{k=1}^{m}\left(a_{k}+b_{k} \sqrt{d}\right)^{2}$ with $a_{k}, b_{k} \in$ $\mathbb{Q}\left(x_{1}, \ldots, x_{n}\right)$. Since $f$ has rational coefficients, by averaging we get

$$
f=\frac{1}{2} \sum_{k=1}^{m}\left(a_{k}+b_{k} \sqrt{d}\right)^{2}+\left(a_{k}-b_{k} \sqrt{d}\right)^{2}=\sum_{k=1}^{m}\left(a_{k}^{2}+d b_{k}^{2}\right)
$$

It remains to write $d$ as a sum of $l \leq 4$ squares of rationals, and we get that $f$ is a sum of at most $(1+l) \cdot m$ squares in $\mathbb{Q}\left(x_{1}, \ldots, x_{n}\right)$.

As another illustration, we apply our method to [3, Example 1.7] :

Example 3.4. Consider the polynomial

$$
f=3-12 y-6 x^{3}+18 y^{2}+3 x^{6}+12 x^{3} y-6 x y^{3}+6 x^{2} y^{4}
$$

which is the following sum of squares

$$
f=\left(x^{3}+\alpha^{2} y+\beta x y^{2}-1\right)^{2}+\left(x^{3}+\beta^{2} y+\gamma x y^{2}-1\right)^{2}+\left(x^{3}+\gamma^{2} y+\alpha x y^{2}-1\right)^{2}
$$

in $\mathbb{Q}(\alpha)[x, y]$ where $\alpha, \beta, \gamma$ are the real roots of the polynomial $u(x)=x^{3}-3 x+1$. 
We do not need to average and directly compute the matrix $G$ and its symmetric LU-decompositon

$$
\left(\begin{array}{rrr}
3 & 0 & 6 \\
0 & 6 & -3 \\
6 & -3 & 18
\end{array}\right)=\left(\begin{array}{rrr}
1 & 0 & 0 \\
0 & 1 & 0 \\
2 & -\frac{1}{2} & 1
\end{array}\right)\left(\begin{array}{lll}
3 & 0 & 0 \\
0 & 6 & 0 \\
0 & 0 & \frac{9}{2}
\end{array}\right)\left(\begin{array}{rrr}
1 & 0 & 2 \\
0 & 1 & -\frac{1}{2} \\
0 & 0 & 1
\end{array}\right)
$$

Because of the relations $\beta=2-\alpha-\alpha^{2}$ and $\gamma=\alpha^{2}-2$, the vector of polynomials $q=\left(q_{0}, q_{1}, q_{2}\right)^{T}$ is $q=\left(x^{3}+2 x y^{2}-1,-x y^{2}, y-x y^{2}\right)^{T}$ and hence

$f=3\left(\left(x^{3}+2 x y^{2}-1\right)+2\left(y-x y^{2}\right)\right)^{2}+6\left(-x y^{2}-\frac{1}{2}\left(y-x y^{2}\right)\right)^{2}+\frac{9}{2}\left(y-x y^{2}\right)^{2}$

a rationnal sum of 3 squares, to compare with the rational sum of 6 squares obtained in [3].

\section{REFERENCES}

[1] M.D. Choi, T.Y. Lam, B. Reznick, Sums of squares of real Polynomials, Proc. Sympos. Pure Math., 58, Part 2, Amer. Math. Soc., Providence, RI, 1995.

[2] S. Basu, R. Pollack, M.F. Roy, Algorithms in Real Algberaic Geometry, Springer

[3] C. J. Hillar, Sums of squares over totally real fields are rational sums of squares, Proc. Amer. Math. Soc. 137 (2009), no. 3, 921-930.

iRmar (CNRS, URA 305), Université de Rennes 1, Campus de Beaulieu, 35042 Rennes Cedex, France

E-mail address: e-mail : ronan.quarez@univ-rennes1.fr 